\title{
Çift Bantlı RF Enerji Hasadı İçin Toplu Eleman Devre Yapıları
}

\author{
Filiz Sari ${ }^{1 *}$ \\ 1*Aksaray Üniversitesi, Mühendislik Fakültesi, Elektrik Elektronik Mühendisliği Bölümü, Aksaray, Türkiye, (ORCID: 0000-0001-8462-175X), \\ filizsari@aksaray.edu.tr
}

(1st International Conference on Applied Engineering and Natural Sciences ICAENS 2021, November 1-3, 2021)

(DOI: 10.31590/ejosat.1010030)

\begin{abstract}
ATIF/REFERENCE: Sari, F. (2021). Çift Bantlı RF Enerji Hasadı İçin Toplu Eleman Devre Yapıları. Avrupa Bilim ve Teknoloji Dergisi, (28), 670-674.

$\ddot{O} \mathbf{z}$

RF enerji hasadı, ortamda bulunan elektromagnetik sinyalleri kullanarak DC enerji üretmektedir. Günümüzde elde edilen enerjiyi arttırmak amacıyla çoklu frekansta RF enerji hasadı çalışmaları yapılmaktadır. Bu çalışmada, çift bantlı RF enerji hasadı için tek empedans uyumlama yapısı ve her bir frekans için ayrı ayrı L tipi empedans uyumlama yapısı çıkış voltaj ve verimleri göz önüne alınarak karşılaştırılmıştır. Simülasyonu yapılan frekans bantları ortamda bulunma yoğunluğu yüksek olan GSM 900 ve GSM 1800 bantlarıdır, bu nedenle $900 \mathrm{MHz}$ ve $1800 \mathrm{MHz}$ frekansları kullanılmış̧ır ve gerilim çoklayıcı olarak iki kademe Dickson gerilim çoklayıcı seçilmiştir. Çoklu bant empedans dönüştürücü için indüktör ve kapasitörlerden oluşan toplu eleman devre yapıları seçilmiştir. Böylece empedans uyumlama devresi çift bantta yükten kaynağa olan yansımaları bastırmıştır ve maksimum güç transferi sağlanmıştır. Bu yapı için maksimum verimler $900 \mathrm{MHz}, 1800 \mathrm{MHz}$ ve çift bant (900\&1800 MHz) için sırasılyla 41.947 \%, 29.259 \%, ve $74.427 \%$ olarak bulunmuştur.
\end{abstract}

Anahtar Kelimeler: RF enerji hasadı, GSM band,, Dickson gerilim çoklayıcı, empedans uyumlama, toplu eleman devreler.

\section{Lumped Element Circuit Structures for Dual-band RF Energy Harvesting}

\begin{abstract}
RF energy harvesting generates DC energy using electromagnetic signals in the environment. Today, multi-frequency RF energy harvesting studies are carried out to increase the energy obtained. In this study, single impedance matching structure for dual band RF energy harvesting and L type impedance matching structure for each frequency are compared considering output voltage and efficiency. The simulated frequency bands are the GSM 900 and GSM 1800 bands, which have a high presence in the environment, so $900 \mathrm{MHz}$ and $1800 \mathrm{MHz}$ frequencies are used and two-stage Dickson voltage multiplier is chosen as the voltage multiplier.Lumped element circuit structures consisting of inductors and capacitors are selected for the multiband impedance converter. Thus, the impedance matching circuit suppress the reflections from the load to the source in the dual band and maximum power transfer was achieved. The maximum efficiencies for this structure are found to be $41.947 \%, 29.259 \%$ and $74.427 \%$ for the $900 \mathrm{MHz}, 1800 \mathrm{MHz}$ and dual bands (900 \& $1800 \mathrm{MHz}$ ), respectively.
\end{abstract}

Keywords: RF energy harvesting, GSM band, Dickson Voltage multiplier, impedance matching, lumped elements.

\footnotetext{
*Sorumlu Yazar: filizsari@aksaray.edu.tr
} 


\section{Giriş}

Günümüzde, enerjiye olan ihtiyacın artmasıyla birlikte güneş, rüzgâr, titreşim ve elektromanyetik dalgalar gibi kaynaklardan enerji hasadı yapılmaya başlanmıştır. Bu kaynaklardan elde edilen güç yoğunluğu enerji kaynağına göre değişiklik göstermektedir. Güneş enerjisi en yüksek güç yoğunluğuna sahip enerji türüdür. Ancak güneş enerjisinden sadece güneş mevcut olduğunda yararlanılır. Termal enerji termoelektrik malzemedeki sıcaklık farkından elde edildiğinden sıcaklık farkının olmadığı durumlarda faydalanılamamaktadır. Titreşimden oluşan enerji ise uygulanan mekanik titreşimlerin şiddetine göre değişmekte ve titreşimlerin olmadığı durumlarda enerji üretilememektedir (Akhtar \& Husain, 2015; Selim et al., 2021; Tran et al., 2017).

Cep telefonu, Wi-Fi gibi kablosuz haberleşme cihazlarının kullanımındaki büyük artışa paralel olarak ortamda mevcut olan elektromanyetik dalgaların varlığı da önemli ölçüde artmıştır. RF enerji hasad1, ortamda az miktarda bulunan enerjinin toplanarak kullanılabilir elektrik enerjisine dönüştürülmesidir. RF enerji hasad1, kablosuz enerji transfer tekniklerinden biridir ve $10 \mathrm{kHz}-$ $300 \mathrm{GHz}$ frekans aralığındaki elektromanyetik sinyallerini içermektedir (Lu et al., 2015; Mantiply et al., 1997).

Bir RF enerji hasatlama devresi genel olarak anten, empedans uyumlama, gerilim çoklayıcı ve enerji depolama bölümlerinden oluşmaktadır. Ortamki RF sinyalleri anten yardımıyla toplanır, gerilim çoklayıcı ile alınan alternatif akım sinyali doğrultulur ve doğru akım (DC) sinyaline dönüştürülür (Sari \& Uzun, 2019). Maksimum güç transferinin sağlanması ve verimi arttırmak için anten ile gerilim çoklayıcı arasında empedans uyumlama devresinin tasarımı RF enerji hasadında önemlidir (Agrawal et al., 2014; Al-Azawy \& Sari, 2019; Li et al., 2021).

Çoklu band enerji hasadında (Agrawal et al., 2018; Keyrouz et al., 2013; Tafekirt et al., 2020; Ozkaya \& Seyfi, 2015; Yalçın \& Sari, 2021)'de belirtildiği gibi farklı yöntemler kullanılmaktadır. Bunlar, her bir frekans için empedans uyumlanmasının ayrı ayrı yapılarak gerilim çoklayıcı devrelerinin birleştirilmesi veya hasadı yapılacak frekanslar için tasarlanan tek bir empedans uyumlama devresinin gerilim çoklayıcı devresine uygulanmasidir.

Bu çalışmada GSM-900 ve GSM-1800 bantları için $900 \mathrm{MHz}$ ve $1800 \mathrm{MHz}$ frekansları incelenmiştir ve gerilim çoklayıcı olarak Dickson gerilim çoklayıcısı seçilmiştir. Çift band RF enerji hasadında toplu öğeli devre yapıları (lumped elements) iki frekansta da empedans uyumlama yapabilecek şekilde (Y. Liu et al., 2012)'deki matematiksel formülüzasyonlar yardımı ile hesapland1. Elde edilen sonuçlar anten girişleri ayrı, her bir frekans için L tipi empedans uyumlama yapılan yap1 ile karşılaştırıldı. Simülasyonlar Advance Design System (ADS) 2017 programı kullanılarak yapılmıştır.

\section{Materyal ve Metot}

\subsection{Gerilim Çoklayııı}

Gerilim çoklayıcılar, antenden alınan alternatif akımı doğru akıma çevirmek ve alınan sinyali yükseltmek için kullanılır. N kademe Dickson gerilim çoklayıcı devresi Şekil 1.'de sunulmuştur.

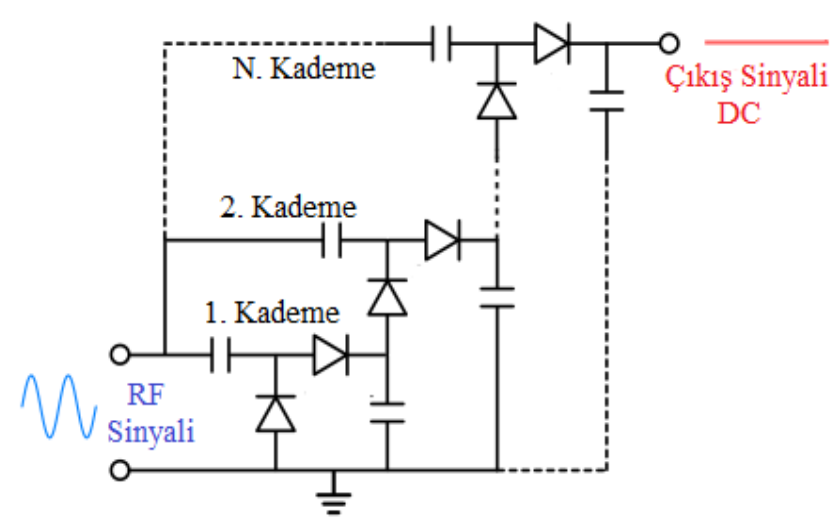

Şekil 1. N Kademe Dickson Gerilim Çoklayıcı

Şekil 1'de 1. kademe temel gerilim çoklayıcı devresidir, iki diyot ve iki kapasitörden oluşmaktadır. 2. kademeden itibaren her kademedeki paralel kapasitörler devrenin toplam empedansını azaltır (Chaour et al., 2017; Di Cataldo \& Palumbo, 1996; Sari \& Uzun, 2019).

RF enerji hasadında, yüksek anahtarlama hızı, düşük ileri gerilim, düşük substrat kaçağı ve nispeten düşük bağlantı kapasitansına sahip olmaları nedeniyle Schottky diyotlar kullanılır, bu çalışmada Avago Technologies'e ait HSMS-2852 Schottky diyot seçilmiştir (Al-Azawy \& Sari, 2019).

\subsection{Empedans Uyumlama}

Kaynak empedansı ve yük empedansı eşit olmadığında, alınan sinyal yükten kaynağa doğru yansıyacaktır. Bu nedenle, alınan sinyalde güç kaybı olacaktır. Empedans uyumlama devresi ile yük empedansının kaynak empedansı ile dengelenmesi sağlanır ve böylece bu tür bir güç kaybı önlenir. Yani, bu empedansların eşitlenmesi yükten kaynağa yansıyan sinyalleri önler böylece en yüksek güç aktarımı kaynaktan yüke yapılabilir (Pozar, 2011; Rhea, 2006). Empedans uyumu, hasat sisteminin önemli bir bileşenidir. L tipi, T tipi ve $\pi$ tipi empedans eşleme ağları empedans eşleme ağ topolojilerinden bazılarıdır (Agrawal et al., 2014). Şekil 2'de 2 kademe Dickson gerilim çoklayıcı için $1800 \mathrm{MHz}$ frekansinda L tipi empedans uyumlama devresi ve Smith-chart gösterimi sunulmuştur.

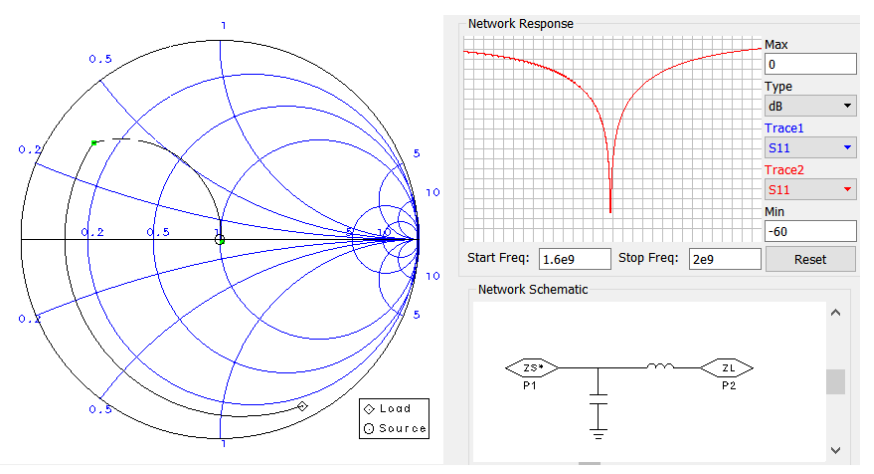

Şekil 2. Tek-band empedans uyumlama örneği

Çok bantlı RF enerji hasadında empedans uyumlamada, her bir bant ayrı ayrı empedans uyumlama yapılarak birleştirilebileceği gibi (Agrawal et al., 2018; Keyrouz et al., 2013; Tafekirt et al., 2020; Uzun, 2015; Yalçın \& Sari, 2021) her banda uygun tepkiyi veren tek bir devre tasarlanabilir (Kim et al., 2013; J. Liu et al., 2020; Y. Liu et al., 2012; Papadopoulou et al., 2020; Saxena et al., 2019), Şekil 3. 


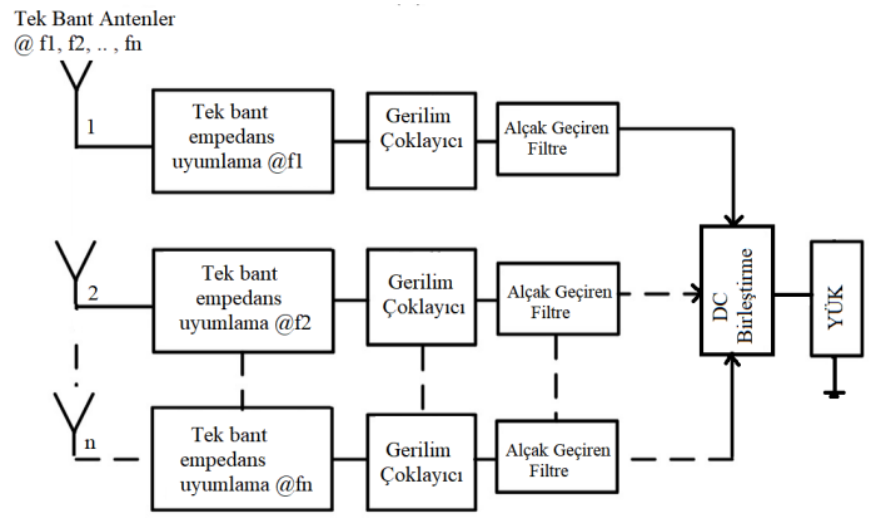

(a)

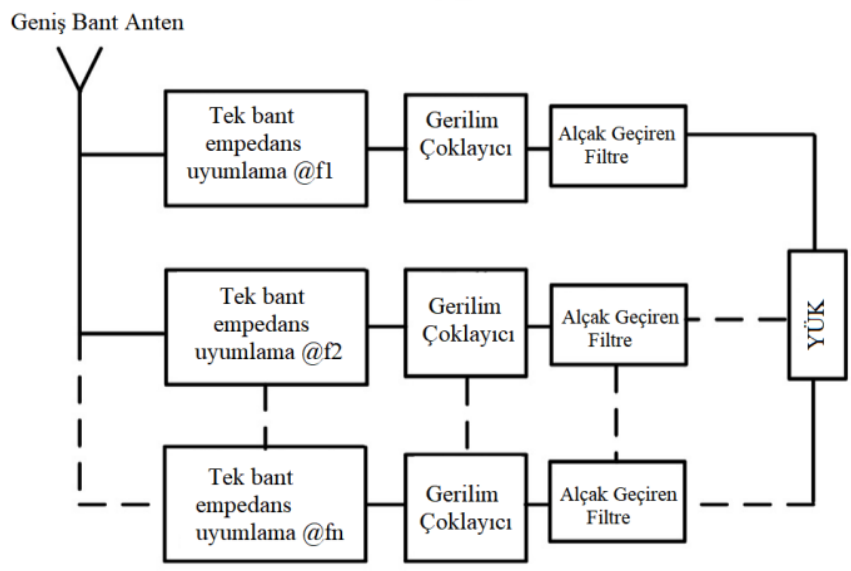

(b)

Çoklu / Geniș bant Anten

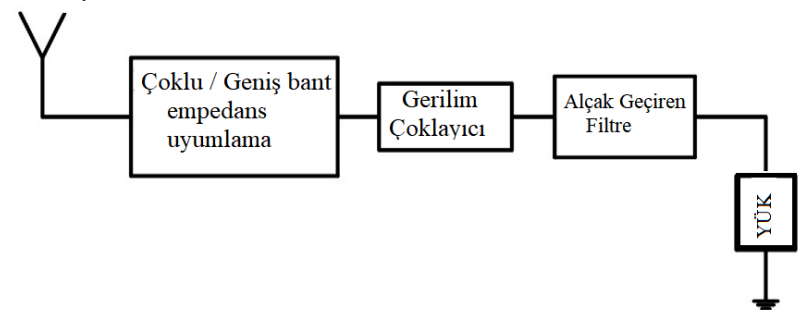

(c)

Şekil 3. Çok bantlı RF enerji hasadı yapıları (a) tek bantlı anten ve tek bantlı empedans uyumlama kullanan (b) geniş bantlı bir anten ve tek bantl empedans uyumlama kullanan ve (c) çok bantll/geniş bant anten ve tek empedans uyumlama kullanan (Agrawal et al., 2018).

Bu çalışmada, Şekil 3 (c)'de gösterilen yapı incelenmiş ve Şekil 3 (a)'daki yapı ile karşılaştırılmıştır. Karşılaştırmalar 2 kademe Dickson gerilim çoklayıcı için yapılmıştır.

Şekil 3 (c)'deki empedans uyumunu sağlamak için (Y. Liu et al., 2012)'de matematiksel ifadeleri verilen indüktör ve kapasitörlerden oluşan, toplu öğeli devre (lumped circuit), çoklu bant empedans dönüştürücü hesaplanarak simülasyonu yapilmıştır.

\section{Araştırma Sonuçları ve Tartışma}

\subsection{Araştırma Sonuçları}

Çalışmada 2 aşamalı Dickson gerilim çoklayıcıda kullanılan kapasitör değerleri $100 \mathrm{pF}$ ve yük direnci $20 \mathrm{k} \Omega$ seçilmiştir. İlk aşamada empedans uyumlama olmadığı durum için $900 \mathrm{MHz}$ ve $1800 \mathrm{MHz}$ için Smith-chart grafiği kullanılarak empedans değerleri bulunmuştur, Şekil 4.

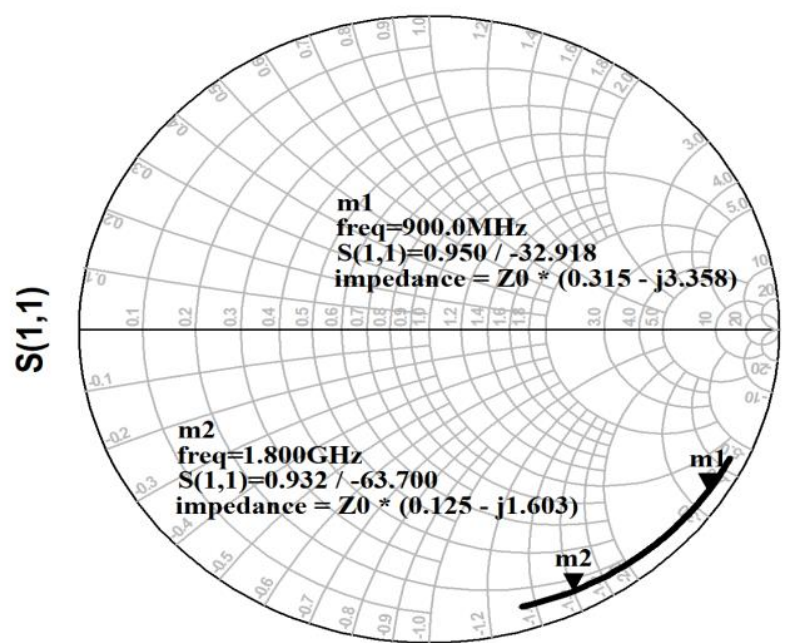

freq $(700.0 \mathrm{MHz}$ to $2.100 \mathrm{GHz})$

Şekil 4. Dickson gerilim çoklayıcı (a) devresi (b) Smith-chart gösterimi

Simülasyonu yapılan devrenin istenilen frekanslar için empedans değerleri Şekil 4'te gösterilmiştir, burada Z0 anten empedansını ifade etmektedir ve tasarımda $50 \Omega$ olarak alınmıştır.

$900 \mathrm{MHz}$ ve $1800 \mathrm{MHz}$ frekansları için bulunan empedans değerleri, (Y. Liu et al., 2012)'de belirtilen C durumuna uymaktadır. Matlab ortamında empedans uyumlama devresinin indüktör ve kapasitör değerleri hesaplanmıştır. Yapılan hesaplamalar sonucunda 2 kademeli Dickson gerilim çoklayıcı için elde edilen empedans uyumlama devresi ve yansıma katsayısı Şekil 5 ve 6'da sunulmuştur.

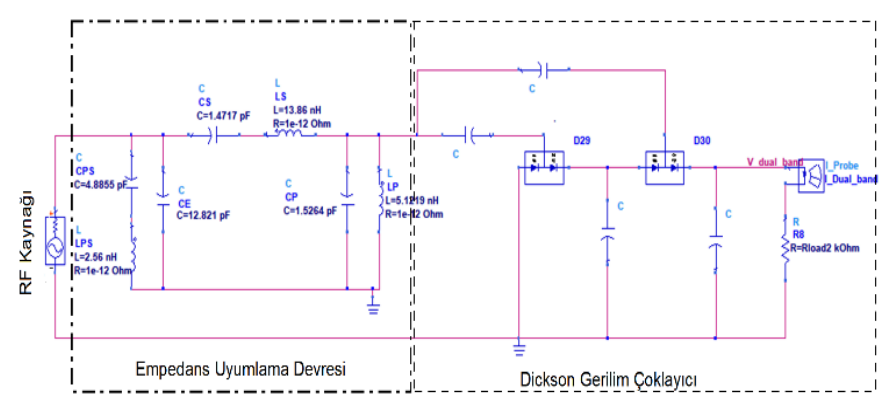

Şekil 5. Çift bantlı bütünleşik empedans uyumlama yapılmış devre

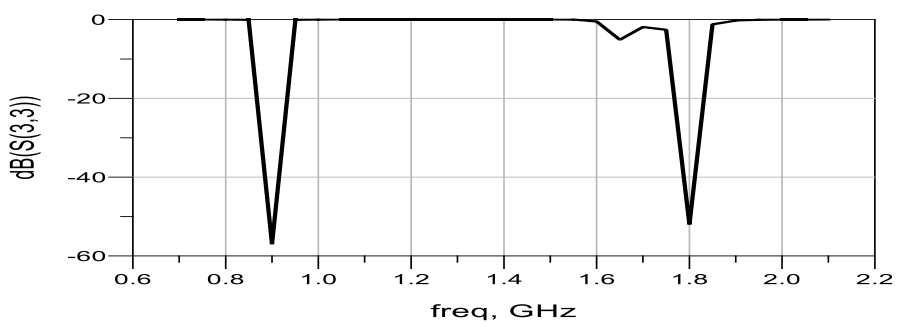

Şekil 6. Empedans uyumlama yapılan devrenin yansıma katsayıs

Şekil 6'de 900 MHz ve 1800 MHz için yükten kaynağa doğru olan yansımalar bastırılmıştır, böylece maksimum güç transferi gerçekleştirilebilecektir. Şekil 7'de çift bantlı bütünleşik empedans uyumu yapılmış devreye sadece $900 \mathrm{MHz}$, sadece 1800 
$\mathrm{MHz}$ ve her iki bant uygulanarak çıkış voltajı ve verimi analiz edilmiştir. RF enerji hasadında verim çıkış gücünün giriş gücüne oranıdır ve $\%$ ile ifade edilir.

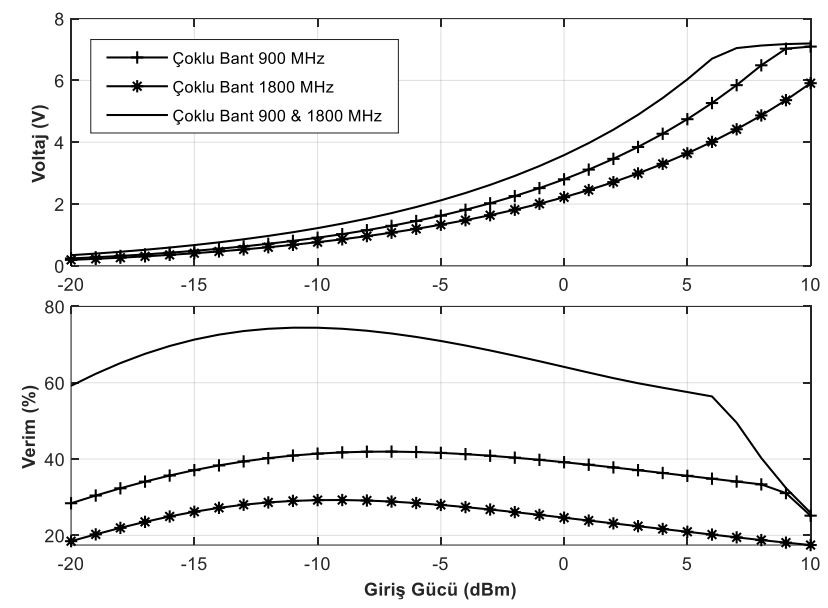

Şekil 7. Çok bantlı tek empedans uyumlu RF enerji hasadı çıkış voltaj ve verimi

Şekil 7'de giriş gücü -20 ile $10 \mathrm{dBm}$ aralığında alınmıştır. Çıkış voltajında, giriş gücü - $10 \mathrm{dBm}$ 'e kadar belirgin bir farklılık gözlenmemekle beraber - $10 \mathrm{dBm}$ 'den daha yüksek giriş gücünde en yüksek voltaj $900 \& 1800 \mathrm{MHz}$ beraber uygulandığında elde edilmiştir. $0 \mathrm{dBm}$ giriş gücü için $900 \mathrm{MHz}, 1800 \mathrm{MHz}$ ve $900 \& 1800 \mathrm{MHz}$ için çıkış voltajları sırasıyla $2.8 \mathrm{~V}, 2.2 \mathrm{~V}$ ve 3.6 $\mathrm{V}$ olarak gözlemlenmiştir. Verim incelendiğinde ise $900 \mathrm{MHz}$ için en yüksek verim - 7 dBm'de $41.947 \%, 1800 \mathrm{MHz}$ 'de - 9 dBm'de $29.259 \%$, her iki frekans uygulandığında ise - 11 dBm'de 74.427 \% olarak bulunmuştur. Bu sayede düşük giriş güçlerinde verim arttırılmıştır.

Her bir frekans için ayrı ayrı L tipi empedans uyumlama yapılarak birleştirilen devre ve yansıma katsayıları Şekil 8'de sunulmuştur. $\mathrm{Bu}$ yöntemde her bir frekans için empedans uyumlama devreleri ayridır.
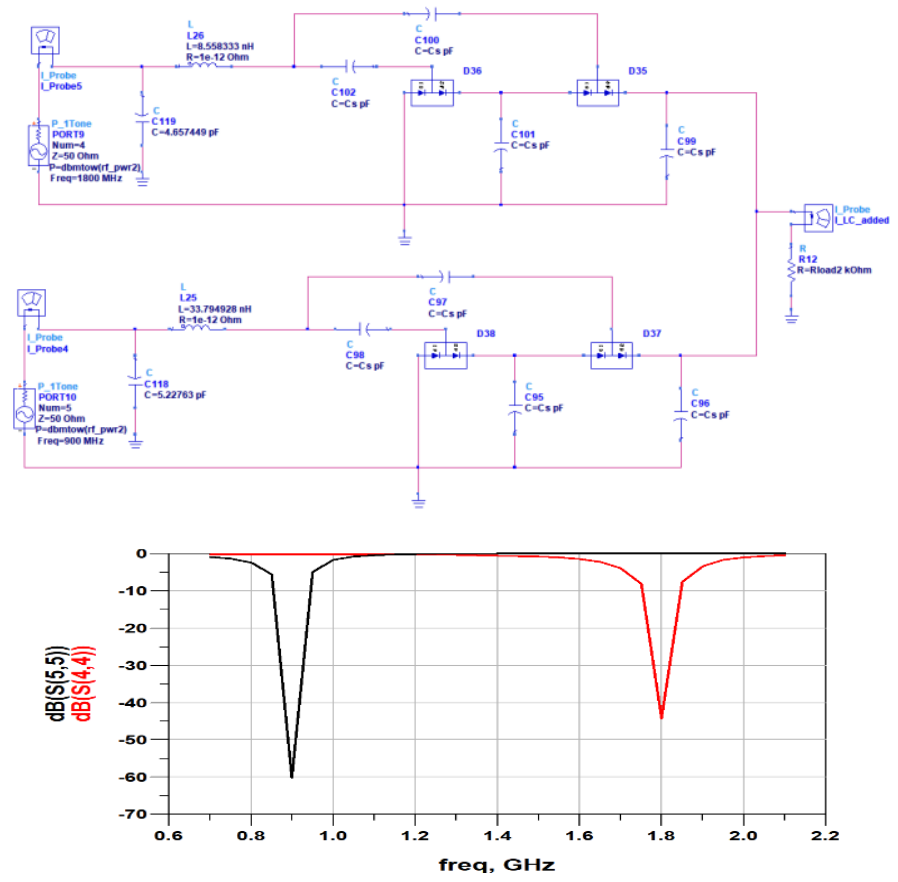

Şekil 8. Çok bantlı ayrı ayrı empedans uyumlama yapılmış devre ve her bir frekans için yansıma katsayısı

e-ISSN: 2148-2683
İki kademeli Dickson gerilim çoklayıcı için, tek empedans uyumlama ve frekansa bağlı ayrı L tipi empedans uyumlamalı RF enerji hasadı çıkış voltaj ve verim sonuçları Şekil 9'da sunulmuştur.

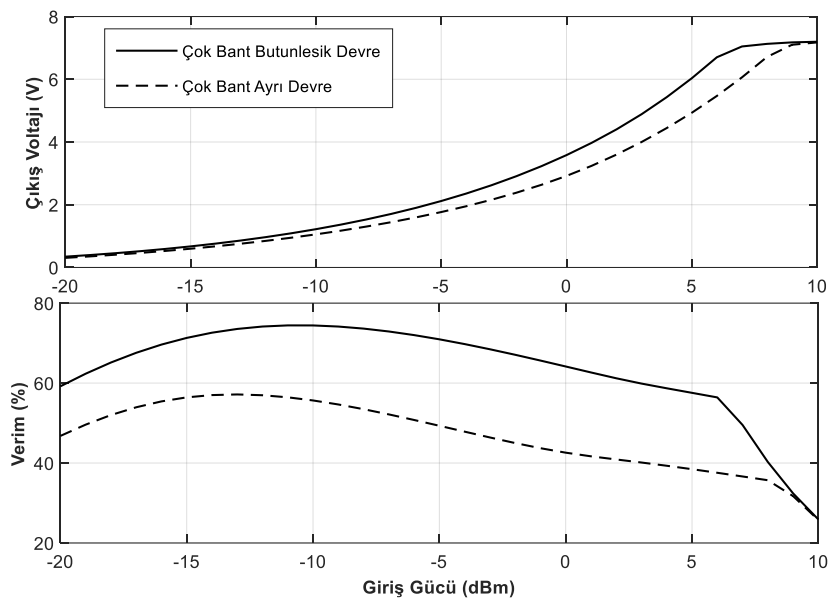

Şekil 9. Çok bantlı empedans uyumlama dereleri için voltaj ve verim değişimi

Şekil 9'da giriş gücüne bağlı olarak çıkış voltajlarında - 10 $\mathrm{dBm}$ 'e kadar belirgin bir farklılık gözlemlenmemiştir, $0 \mathrm{dBm}$ giriş gücü için tek empedans uyumlama için $3.582 \mathrm{~V}$, her bir bant için L tipi empedans uyumlama için $2.919 \mathrm{~V}$ çıkış elde edilmiştir. Verim grafiğinde ise tek empedans uyumlamada $-11 \mathrm{dBm}$ giriş için $74.427 \%$, her bir bant için L tipi empedans uyumlamada toplamda $-13 \mathrm{dBm}$ de $57.159 \%$ verim elde edilmiştir.

\subsection{Tartışma}

RF enerji hasadı, ortamda bulunan elektromagnetik sinyalleri kullanarak enerji üretmektedir. Bu çalışmada, ikili RF bantı için ADS programı kullanılarak çıkış voltaj ve verimleri incelenmiştir. Çift bantlı, 900 \& $1800 \mathrm{MHz}$ için RF enerji hasadı uygulamaları incelendiğinde, (Ho et al., 2016; Pham \& Pham, 2013; Zeng et al., 2018), verim $62 \%$ ile $80 \%$ aralığında değişmektedir. Bu farklılıkların nedeni gerilim çoklayıcının kademe sayısı, kullanılan diyot türü ve farklı empedans uyumlama türleridir.

$\mathrm{Bu}$ çalışmada iki farklı tür çoklu RF enerji hasadının karşılaştırılması için gerilim çoklayıcıların kademeleri aynı seçilmiştir. İki frekans için L tipi empedans uyumlama ile birleştirilen yapının verim değerleri, iki frekans için tek uyumlama devresi tasarlandığı duruma göre daha düşük çıkmıştır. Düşük giriş güçlerinde tek empedans uyumlama devresi yaklaşık olarak $20 \%$ verim kazancı sağlamıştır. Lumped elemanlar kullanılarak tek empedans uyumlama tekniğinin veriminin 74.427 \%’ye kadar çıktığı gözlemlenmiştir.

\section{Sonuç}

$\mathrm{Bu}$ çalışmada ikili bant RF enerji hasadı için iki farklı empedans uyumlama devre türü karşılaştırılmıştır. Kullanılan elemanlar lumped elemanlardır. Seçilen frekanslar için kaynaktan yüke yansımaları bastıran empedans uyumlama devresi (Y. Liu et al., 2012)'deki denklemler yardımıyla bulunmuştur ve verim 74.427\%' ye kadar yükseltilmiştir. İki bant ve her bir frekans için $\mathrm{L}$ tipi empedans uyumlama yapıldığında ise verim $57.159 \%$ olarak bulunmuştur. İki frekansın tek bir empedans uyumlama ile birleştirildiği yapı düşük giriş gücü seviyelerinde L tipi empedans uyumlamaya göre $20 \%$ iyileştirme sağlamıştır. 


\section{Kaynakça}

Agrawal, S., Pandey, S. K., Singh, J., \& Parihar, M. S. (2014). Realization of efficient RF energy harvesting circuits employing different matching technique. Fifteenth International Symposium on Quality Electronic Design, c, 754-761.

Agrawal, S., Parihar, M. S., \& Kondekar, P. N. (2018). A quadband antenna for multi-band radio frequency energy harvesting circuit. AEU - International Journal of Electronics and Communications, 85(October 2017), 99-107.

Akhtar, F., \& Husain, M. (2015). Energy replenishment using renewable and traditional energy resources for sustainable wireless sensor networks: A review. Renewable and Sustainable Energy Reviews, 45, 769-784.

Al-Azawy, M. M., \& Sari, F. (2019). Analysis of Dickson Voltage Multiplier for RF Energy Harvesting. 2019 1st Global Power, Energy and Communication Conference (GPECOM), July, 10-14.

Chaour, I., Fakhfakh, A., \& Kanoun, O. (2017). Enhanced passive RF-DC converter circuit efficiency for low RF energy harvesting. Sensors (Switzerland), 17(3), 1-14.

David M. Pozar. (2011). Microwave Engineering. Wiley.

Di Cataldo, G., \& Palumbo, G. (1996). Design of an Nth order Dickson voltage multiplier. IEEE Transactions on Circuits and Systems I: Fundamental Theory and Applications, 43(5), 414-418.

Ho, D., Kharrat, I., Vuong, V. N. T., Nguyen, Q., \& Le, M. (2016). Dual-Band Rectenna for Ambient RF Energy Harvesting at GSM $900 \mathrm{MHz}$ and $1800 \mathrm{MHz} .1,306-310$.

Keyrouz, S., Visser, H. J., \& Tijhuis, A. G. (2013). Multi-band Simultaneous Radio Frequency Energy Harvesting. 2013 7th European Conference on Antennas and Propagation (EuCAP), Eucap, 3058-3061.

Kim, P., Chaudhary, G., \& Jeong, Y. (2013). A Dual Band RF Energy Harvesting Using Frequency Limited Dual-Band Impedance Matching. Progress In Electromagnetics Research, 141(July), 443-461.

Li, P., Long, Z., \& Yang, Z. (2021). RF Energy Harvesting for Batteryless and Maintenance-Free Condition Monitoring of Railway Tracks. IEEE Internet of Things Journal, 8(5), 35123523.

Liu, J., Huang, M., \& Du, Z. (2020). Design of Compact DualBand RF Rectifiers for Wireless Power Transfer and Energy Harvesting. IEEE Access, 8, 184901-184908.

Liu, Y., Zhao, Y.-J., \& Zhou, Y. (2012). Lumped Dual-Frequency Impedance Transformers for Frequency-dependent Complex Loads. Progress In Electromagnetics Research, 126(December 2011), 121-138.

Lu, X., Wang, P., Niyato, D., Kim, D. I., \& Han, Z. (2015). Wireless Networks With RF Energy Harvesting: A Contemporary Survey. IEEE Communications Surveys \& Tutorials, 17(2), 757-789.

Mantiply, E. D., Pohl, K. R., Poppell, S. W., \& Murphy, J. A. (1997). Summary of measured radio frequency electric and magnetic fields $(10 \mathrm{kHz}$ to $30 \mathrm{GHz})$ in the general and work environment. Bioelectromagnetics, 18(8), 563-577.

Ozkaya, U., \& Seyfi, L. (2015). Dimension optimization of microstrip patch antenna in $\mathrm{X} / \mathrm{Ku}$ band via artificial neural network. Procedia-Social and Behavioral Sciences, 195, 2520-2526.

Rhea, R. (2006). The Yin-Yang of Matching: Part 1 - Basic Matching. Matching Networks.
Papadopoulou, M. S., Boursianis, A. D., Skoufa, A., Volos, C. K., Stouboulos, I. N., Nikolaidis, S., \& Goudos, S. K. (2020). Dual-Band RF-to-DC Rectifier with High Efficiency for RF Energy Harvesting Applications. 2020 9th International Conference on Modern Circuits and Systems Technologies (MOCAST), 1-4.

Pham, B. L., \& Pham, A. V. (2013). Triple bands antenna and high efficiency rectifier design for RF energy harvesting at 900, 1900 and $2400 \mathrm{MHz}$. IEEE MTT-S International Microwave Symposium Digest, 13-15.

Sari, F., \& Uzun, Y. (2019). a Comparative Study: Voltage Multipliers for Rf Energy Harvesting System. Commun.Fac.Sci.Univ.Ank.Series A2-A3, 61(1), 12-23.

Saxena, A., Banerjee, D., Hashmi, M., \& Auyenur, M. (2019). A Dual-Band Impedance Transfomer for Matching Frequency Dependent Complex Source and Load Impedances. 2019 15th Conference on Ph.D Research in Microelectronics and Electronics (PRIME), July, 173-176.

Selim, K. K., Wu, S., Saleeb, D. A., \& Ghoneim, S. S. M. (2021). A Quad-Band RF Circuit for Enhancement of Energy Harvesting. Electronics, 10(10), 1160.

Tafekirt, H., Pelegri-Sebastia, J., Bouajaj, A., \& Reda, B. M. (2020). A Sensitive Triple-Band Rectifier for Energy Harvesting Applications. IEEE Access, 8, 73659-73664.

Tran, L. G., Cha, H. K., \& Park, W. T. (2017). RF power harvesting: a review on designing methodologies and applications. Micro and Nano Systems Letters.

Yalçın, A. B., \& Sari, F. (2021). Efficiency Analysis For Triple Band Rf Energy Harvesting. Aksaray University Journal of Science and Engineering, 5(1), 36-45.

Zeng, M., Li, Z., Andrenko, A. S., Zeng, Y., \& Tan, H. (2018). Research Article A Compact Dual-Band Rectenna for GSM900 and GSM1800 Energy Harvesting. 2018, 1-10. 